\title{
PROPUESTA Y BASES JURÍDICAS PARA LA ELABORACIÓN DE UN PROGRAMA DE PAGO POR SERVICIOS AMBIENTALES PARA LA LAGUNA DE SONTECOMPAN, MÉXICO
}

Tania García López ${ }^{1}$

Universidad Veracruzana

Mayra Lizeth Gudiño Anaya ${ }^{2}$

Universidad Veracruzana, México

\section{RESUMEN}

En este trabajo hacemos una propuesta para la elaboración de un programa de pago por servicios ambientales (PSA) para la laguna de Sontecompan, Veracruz, México.

Se definen y explica los distintos pasos a seguir para la elaboración de dicho programa, se construyen escenarios hipotéticos de conservación y se identifican, tanto a los beneficiados como a los obligados a pagar por estos servicios.

Este tipo de programas todavía no cuentan con unas bases jurídicas muy claras para su desarrollo y éstas deberán estar en relación con el nivel de gobierno (federal, estatal o municipal) en el cual se encuentren los beneficiados directos por los servicios ambientales en cuestión.

Otro aspecto jurídico a considerar y que se analiza en este artículo es la celebración de uno o varios contratos que reflejen las obligaciones de las partes. Hay una amplia gama de opciones disponibles y los detalles del contrato dependerán, en gran medida, de la naturaleza de las partes. Las transacciones de PSA a menudo implican un único comprador y múltiples vendedores. Otros tipos de relaciones pueden incluir a un solo comprador y un único vendedor o bien a un solo comprador y varios vendedores, agrupados a través de una cooperativa u otra institución que se ocupe de los problemas colectivos.

1 Doctora en Derecho ambiental por la Universidad de Alcalá de Henares, España. Investigadora en Universidad Veracruzana. Investigadora Nacional nivel 2, CONACYT, México. ORCID: https:// orcid.org/0000-0002-9540-3691 / e-mail: tgar70@gmail.com

2 Ingeniero ambiental por la Universidad Veracruzana, México. E-mail: mayra_ga15@hotmail.com 
En definitiva, este trabajo propone un instrumento económico en materia ambiental: pago por servicios ambientales, financiado por el sector privado y subraya los aspectos jurídicos para su correcta puesta en marcha.

Palabras clave: instrumentos económicos en material ambiental; pago por servicios ambientales.

\section{PROPOSAL AND LEGAL BASES FOR THE ELABORATION OF A PAYING FOR ECOSYSTEM SERVICES PROGRAM FOR SONTECOMPAN LAGOON, MÉXICO}

\section{ABSTRACT}

In this paper we make a proposal for the development of a paying for ecosystem services (PSA) Program for Sontecomapan lagoon, Veracruz, Mexico.

The different steps to be followed for this proposal are defined and explained; hypothetical conservation scenarios are constructed and both the beneficiaries and those obligated to pay for these services are identified. These types of programs do not yet have very clear legal bases for their development and these bases should be related to the level of government (federal, state or municipal) in which the direct beneficiaries are located. Another legal issue which is considered and analyzed in this article is the building of one or several contracts that reflect the obligations of the parties. There is a wide range of available options for this purpose and the details of the contract will largely depend on the nature of the parties. PSA transactions often involve a single buyer and multiple sellers. Other types of relationships can include a single buyer and seller or a single buyer and several sellers grouped through a cooperative or another institution that deals with collective problems.

In short, this work proposes an economic environment instrument: paying for ecological services, financed by the private sector and it emphasizes the legal aspects for its proper implementation.

Keywords: economic environmental instruments; paying for ecosystem services. 


\section{INTRODUCCIÓN}

Las sociedades obtienen una amplia variedad de beneficios de los ecosistemas, conocidos como servicios ambientales, entre los que se encuentran los alimentos, las medicinas, las materias primas para la construcción, así como el agua que bebemos o utilizamos para diferentes fines y son los que proporcionan, en gran medida, el sustento de las sociedades humanas y de las economías mundiales (FREGOSO et al., 2006, p. 29-46).

El crecimiento poblacional y la expansión urbana son varias de las razones por las cuales, en los últimos años, se ha provocado un grave impacto ambiental en los manglares, que se refleja en la contaminación del aire, del agua y del suelo. Además de lo anterior, se ha producido una importante pérdida de la diversidad biológica, el desequilibrio de los ciclos naturales y el cambio de uso del suelo, con efectos secundarios como la erosión, la salinización y la desertificación (PEREVOCHTCHIKOVA Y OCHOA TAMAYO, 2012).

Uno de los temas actuales más apremiantes en materia ambiental es el considerable proceso de deterioro que presentan los ecosistemas a nivel mundial. Esta pérdida afecta de manera negativa a la capacidad de los ecosistemas para realizar sus funciones y procesos ecosistémicos, relacionados con la regulación hidrológica y, por lo tanto, su capacidad de proveer servicios ambientales. (PEREVOCHTCHIKOVA Y OCHOA TAMAYO, 2012).

En México, se estima que se han perdido aproximadamente un $65 \%$ de los manglares y también que la tasa de deforestación es del 5\% anual (OLGUÍN et al., 2007, p. 139-154). Debido a todo lo anterior, se han identificado incentivos económicos como el pago por servicios ambientales (PSA), como una posible herramienta para prevenir el deterioro ambiental, mantener y mejorar la calidad de los manglares y, por ende, de la vida humana. Esta iniciativa tiene como fin aminorar y prevenir los problemas ambientales, a través de la participación activa de los beneficiarios y proveedores de los servicios ambientales. El PSA plantea que los propietarios o poseedores de las tierras sean retribuidos por los servicios que éstas generan, conciliando así sus intereses con los de la sociedad a la que beneficia (FREGOSO et al., 2006, p. 29-46), Además, son una estrategia para que los poseedores de los recursos naturales, principalmente de países no desarrollados, se motiven a conservar en buen 
estado los ecosistemas. (MARTÍNEZ CRUZ et al., 2010, p. 549-556).

La Convención de Ramsar o Convención sobre los Humedales es un "tratado intergubernamental aprobado el 2 de febrero de 1971 en la localidad Iraní de Ramsar, situada a orillas del mar Caspio que refleja el énfasis puesto inicialmente en la conservación y el uso racional de los humedales sobre todo como hábitat de tales aves" (SECRETARÍA DE LA CONVENCIÓN DE RAMSAR, 2013). Los sitios Ramsar son "humedales que cumplen una serie de criterios establecidos para poder adicionarse a la Lista de Humedales de Importancia Internacional. Estos Criterios se llaman Criterios de Importancia Internacional y han sido adoptados por la cuarta, sexta y séptima Reuniones de la Conferencia de las Partes Contratantes de la Convención sobre los Humedales" (RAMSAR, 1987).

El sitio Ramsar, con el nombre de "Manglares y Humedales de la Laguna de Sontecomapan" cumple con los criterios 1,2,3,4,7 y 8 del tratado (ESTEROS, 2003). El primer criterio se refiere a que un humedal podrá ser considerado internacional "si contiene un ejemplo representativo, raro o único de un tipo de humedal natural o casi natural hallado dentro de la región biogeográfica apropiada" (RAMSAR, 1971). El humedal de la laguna cuenta con especies amenazadas como la aguililla negra y en peligro de extinción como el taimán, por ello cumple con el segundo criterio, el cual nos menciona que el humedal debe contar con dichas especies para poder ser considerado de importancia internacional. El tercer criterio se refiere a que será considerado internacional "si sustenta poblaciones de especies vegetales y/o animales importantes para mantener la diversidad biológica de una región biogeográfica determinada "(RAMSAR, 1971) en este caso, en el manglar. El humedal ofrece refugio a miles de especies y con ello cumple el cuarto criterio. El siguiente criterio es el séptimo y lo cumple el humedal, debido a que sustenta gran cantidad de especies "que son representativas de los beneficios y/o los valores de los humedales y contribuye de esa manera a la diversidad biológica del mundo" (RAMSAR, 1971). El último criterio que cumple el humedal es el octavo y es debido a que es fuente de alimentación de especies o "es una zona de desove, un área de desarrollo y crecimiento y/o una ruta migratoria de la que dependen las existencias de peces dentro o fuera del humedal" (RAMSAR, 1971).A pesar de ello, las disposiciones de la Convención no garantizan un nivel de protección suficiente para estos ecosistemas y, por ello, es necesario adoptar medidas que, más allá de las tradicionales regulaciones jurídicas, fomenten una efectiva protección de este ecosistema. 
En este trabajo proponemos la elaboración de un un programa de pago por servicios ambientales para la Laguna de Sontecomapan, México y profundizamos sobre los aspectos jurídicos que debe considerar dicho programa.

\section{OBJETIVO}

Proponer el diseño de un programa de pago por servicios ambientales para la Laguna de Sontecomapan, en México y profundizar sobre los aspectos jurídicos que debe considerar dicho programa.

\section{METODOLOGÍA}

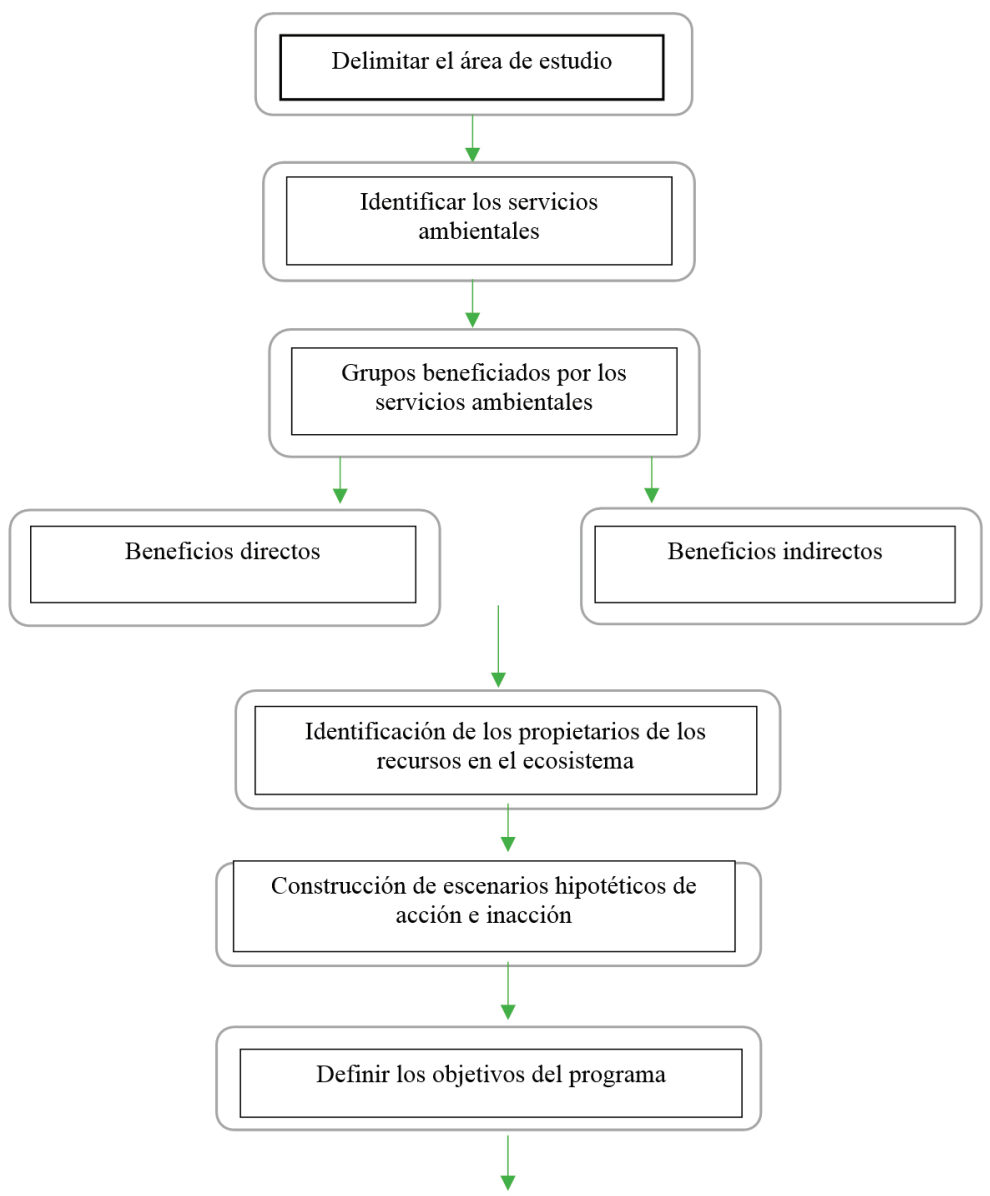




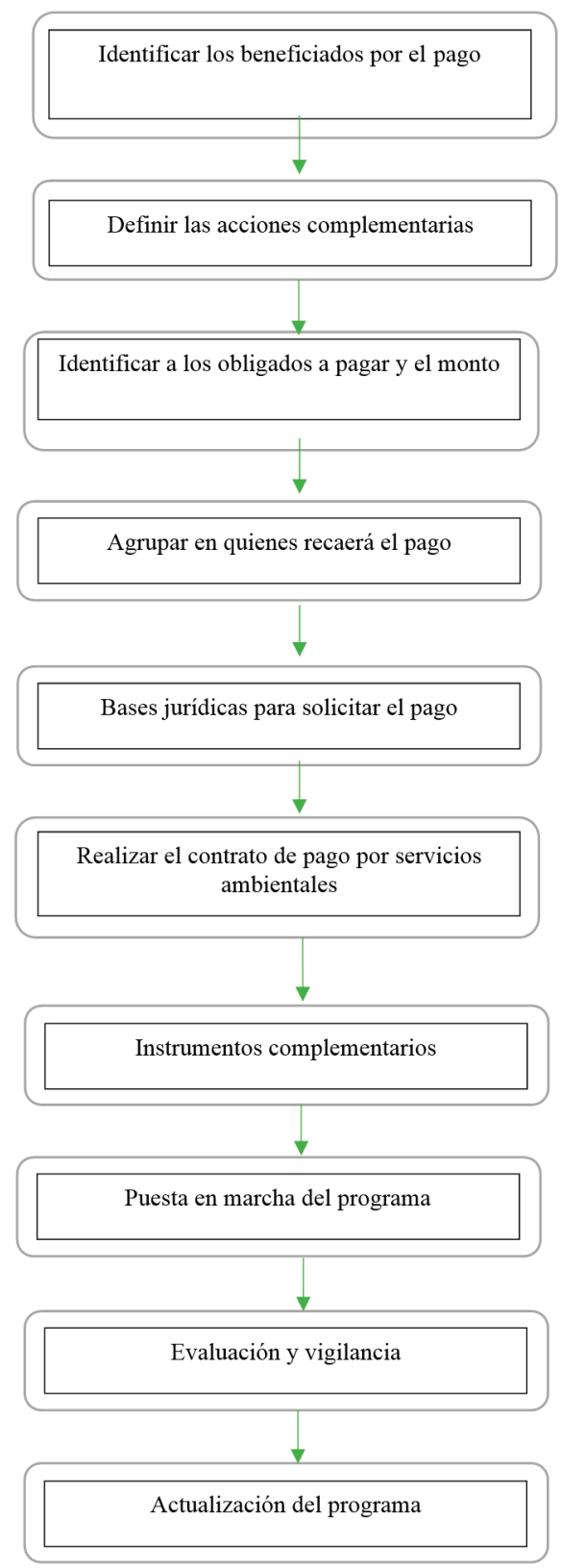


Para el diseño del programa de pago por servicios ambientales se propone la metodología elaborada por Tania García López: García (2018a, p. 147-165) y García (2018b, p. 41-62), la cual identifica los pasos a seguir para la elaboración de este tipo de programas.

\section{PROGRAMA DE PAGO POR SERVICIOS AMBIENTALES PARA LA LAGUNA DE SONTECOMAPAN, MÉXICO}

\subsection{Delimitación de la zona o ecosistema sobre la que se quiere influir}

\subsubsection{Zona de conservación y restauración}

El espacio elegido para las acciones de conservación y/o restauración objeto de esta propuesta es la Laguna de Sontecomapan, que se encuentra en la cuenca hidrológica Río Papaloapan. Esta zona es donde se generan los servicios ambientales que son aprovechados por el hombre, siendo hogar de un gran número de especies de flora y fauna que necesitan protección, así como de manglares y humedales que se encuentran dentro de ésta y que son de gran importancia. Dicha laguna se ubica en la región de los Tuxtlas, al sureste del estado de Veracruz, a $20 \mathrm{~km}$ al Noreste de la ciudad de Catemaco y a dos horas de la ciudad y puerto de Veracruz, por la carretera federal No.180 (ESTEROS, 2003).

Se trata de la zona objetivo o la zona por la cual se diseña el programa. En esta área se encuentran los recursos más vulnerables y asimismo los que brindan un mayor número de servicios ambientales. Es, en definitiva, el ecosistema que justifica el diseño del programa de pago por servicios ambientales (GARCÍA, 2018b, p. 41-62).

\subsubsection{Zona de aplicación del programa}

La zona de aplicación es mucho más amplia que la anterior debido a que es la zona que influencia directamente a la laguna. Abarca la cuenca hidrológica completa llamada Río Papaloapan, que comprende 445 municipios de cuatro estados donde radica una población de 3.3 millones de habitantes (CONAGUA, 2014). Son 161 municipios de Oaxaca, 90 de Puebla, 5 de Hidalgo y 189 de Veracruz (SEFIPLAN, 2011). 


\subsection{Identificación de los servicios ambientales}

A continuación se describen algunos servicios ambientales importantes que provee la laguna:

- Belleza escénica;

- Protección de la diversidad;

- Provisión de agua;

- Crianza de especies y producción de alimentos;

- Protección del suelo;

- Captura de carbono;

- Control de la erosión;

- Protección contra inundaciones;

- Filtrado de aguas residuales;

- Filtro biológico.

\subsection{Grupos beneficiados por los servicios ambientales}

Existe un gran número de beneficiados por los servicios ambientales que provee el manglar y la laguna de Sontecomapan: habitantes de los 6 municipios, habitantes de poblaciones aledañas a la laguna, habitantes de los municipios de la cuenca, habitantes de los municipios del estado, turistas de otros estados y de otros países. Ya sea directa o indirectamente dichos grupos son beneficiados por los servicios que se mencionan más adelante.

Cabe mencionar que, así como algunos servicios ambientales benefician directa o indirectamente a los municipios que dan a la laguna, también lo hacen directamente o indirectamente a los municipios de la cuenca del río Papaloapan, que comprende los 445 municipios y que son los que influencian directamente a la laguna.

\subsection{Beneficios directos vs. beneficios indirectos}

Es muy importante distinguir entre beneficios directos e indirectos, "puesto que es deseable que los servicios ambientales sean pagados, al menos en un principio, por los beneficiados directos, aunque haya otros beneficiados indirectamente por estos servicios. A medida que este tipo de esquemas permeen en una sociedad sería deseable que todos los beneficiados por un servicio ambiental pagasen éste" (GARCÍA, 2018b). 


\subsection{Identificación de los propietarios o poseedores de los recursos contenidos en el ecosistema}

Desde el decreto de Lázaro Cárdenas para dividir a Sontecomapan en ejidos y con la posterior autorización del gobierno para la venta de estos, el área ha cambiado de dueños paulatinamente, "debido a la constante modificación de propietarios de los terrenos privados y a la falta de delimitaciones físicas de estas áreas, es difícil indicar con precisión cómo se encuentran conformadas las propiedades actuales y cuál es la superficie que representan" (TOLEDO et al., 1972, p. 199-237). En términos generales, la tenencia de la tierra se encuentra distribuida en terrenos federales, privados y en ejidos, siendo todos estos eminentemente ganaderos, pero con actividades de recoleccion y aprovechamiento de recursos naturales en los bosques cercanos.

\subsection{Construcción de escenarios hipotéticos de conservación y su relación con los servicios ambientales}

Este paso del programa se centra en la construcción de escenarios hipotéticos de conservación de los recursos de la zona. "Lo más recomendable es establecerlos a mediano o largo plazo, 20 ó 30 años, tiempo que se considera adecuado para observar cambios en un ecosistema con una política de conservación adecuada" (GARCÍA, 2018b, p. 41-62). Se propone la construcción de tres escenarios y la proyección de cada uno para los manglares de la Laguna de Sontecomapan.

\subsubsection{Escenario 1}

En el primer escenario, llamado pesimista, se tomó como base el período de cinco años, del 2005 al 2010, debido a que fue el período en el que se presentó más porcentaje de pérdida del manglar. En el 2005 se tenía una superficie de manglar de 782 ha y un porcentaje de pérdida del 3\% en esos cinco años. Así, para el 2010 se tuvo una superficie final de manglar de 760 ha. (Dichos datos fueron tomados de la ficha de caracterización de CONABIO) (LARA-DOMÍNGUEZ et al., 2009).

Este escenario se realiza con el objetivo de mostrar el lado más extremo que le ocurriría a la Laguna de Sontecomapan en un periodo de 20 años, tomando el porcentaje de pérdida que se tuvo en períodos pasados. Con ello 
nos daríamos cuenta de la pérdida que se tendría de manglar en el periodo del 2018 al 2038 si se sigue la misma gestión de éste. Este escenario supone que los habitantes con terrenos dentro del manglar no realizan ninguna acción por conservarlo y/o protegerlo. Del mismo modo, habitantes de la cuenca y de otros municipios siguen explotando de forma descontrolada los recursos que provee la laguna. Las autoridades no se preocupan por el futuro de la laguna y de los manglares, ni de su posible desaparición por el mal manejo de ésta. Por el contrario, se siguen explotando sus recursos de una manera descontrolada, se hacen cada vez más grandes las amenazas de extracción de madera y deforestación.

Tomando en cuenta el porcentaje de pérdida del 3\% del período preestablecido y siguiendo la mala administración, en el periodo del 2018 al 2038 se tendría una superficie de manglar de aproximadamente 636 ha. Dicho dato se obtuvo de aplicar el porcentaje de pérdida constante de 3\% por cada cinco años. Por lo tanto, bajo este escenario pesimista, se tendría una pérdida de 146 ha. Esta cantidad se calculó restando las 760 ha que había en en primer periodo a las 636 ha del periodo estimado.

\subsubsection{Escenario 2}

En el segundo escenario, llamado intermedio, se tomó el periodo preestablecido de veintinueve años de 1976 al 2005 donde se mostró un porcentaje de pérdida moderado del $2 \%$ con una superficie de manglar de 782 ha, datos obtenidos de la ficha de caracterización de CONABIO (LARA-DOMÍNGUEZ et al., 2009). En dicho período se tuvo un porcentaje de ganancia de manglar del 12\% debido a la rehabilitación ecológica que se tuvo en ese año.

En este escenario la laguna de Sontecomapan sigue estando igual que como se encuentra ahora, su administración es la misma y no se ha hecho nada al respecto. Los habitantes y turistas que la visitan siguen sin tomar en cuenta la importancia de preservar, cuidar y proteger el manglar.

En este escenario se tomó un porcentaje de pérdida calculado a partir del 2\% que se perdió en 29 años en el periodo de 1976 al 2005 en el que se contaba con 782 ha. Dicho porcentaje de pérdida calculado se obtuvo a partir de la división del porcentaje de pérdida de manglar del $2 \%$ entre los años del periodo y con ello se obtuvo el porcentaje de $0.06 \%$ de la superficie. Tomando en cuenta el porcentaje de pérdida calculado anteriormente del periodo preestablecido y siguiendo la misma gestión 
de la laguna en el periodo del 2018 al 2028 se tendría una superficie de manglar de aproximadamente 759 ha; dato que se obtuvo de aplicar el porcentaje de pérdida constante de $0.06 \%$ por cada año. Por lo tanto se tendría una pérdida de 23 ha, esta cantidad resultó de restar las 782 ha del primer periodo a las 759 ha del periodo estimado con el objetivo de realizar un escenario intermedio en la laguna.

\subsubsection{Escenario 3}

En el tercer escenario, llamado optimista, se espera la recuperación del manglar de Sontecomapan. Se gestionará con un programa de pago por servicios ambientales, habrá un mayor nivel de conservación y se tendrá un control de los servicios ambientales que provee la laguna. Al preservar y proteger el ecosistema con dicho programa se ayudaría a que la laguna siga brindando dichos servicios e incluso los amplíe. Se dejarían de explotar los recursos de una manera descontrolada, disminuyendo la extracción de madera y generando reforestación en zonas que lo necesitan, además de acciones de conservación.Tomando en cuenta que en el 2010 se tenía una superficie de manglar de 760 ha, con la aplicación del programa de pago por servicios ambientales se esperaría que se mantuviera la superficie de manglar en la Laguna de Sontecomapan aplicando algunas medidas de reforestación en algunas zonas vulnerables.

\subsection{Objetivos del programa de pago por servicios ambientales}

Para una adecuada construcción del programa de pago por servicios ambientales y una eficiente aplicación, es necesario después de construir los escenarios hipotéticos, el plantear los objetivos claros del programa y estos deben estar en relación con cada servicio ambiental que provee la laguna. Todo esto se hace con el objetivo de poder llegar a preservar y proteger el ecosistema. Al mismo tiempo, se dejarían de explotar los recursos de una manera descontrolada, disminuyendo, así, la extracción de madera y deteniendo de manera significativa la deforestación.

Todos los objetivos planteados son importantes para la elaboración del pago por servicios ambientales debido a que nos sirven para realizar las acciones para la preservación de los servicios ambientales. Para el servicio ambiental de protección contra inundaciones se deberá resguardar la zona en la que se tenga manglar y hacer acciones de reforestación en las 
zonas donde se presente excesiva tala de árboles. Dichas acciones deberán realizarse por ejidatarios, comuneros y posesionarios, así como por las personas dueñas de propiedades privados.

Para abatir el desgaste que se produce en la superficie del suelo y para que se pueda proteger el suelo, se deberán realizar acciones similares al servicio ambiental anterior, reforestando las zonas donde haya habido excesiva tala de árboles y preservando el suelo que se tenga en óptimas condiciones. Dichas acciones deberán ser realizadas por propietarios privados, ejidatarios, comuneros y posesionarios.

Es muy importante el mantener y preservar la belleza de un lugar ,así como su biodiversidad, en parte para que más turistas sigan visitando el sitio y para que se sigan gozando de los servicios que provee. Para ello, se deberán implementar más botes de basuras en la zona de las embarcaciones y recolectar periódicamente la basura en la zona del manglar y en los alrededores de la laguna y seguir reforestando. Para lograr lo anterior es necesario que: gobierno municipal, habitantes del municipio, concesionarios y pescadores realicen esas acciones.

La crianza de especies y la producción de alimentos son servicios ambientales de vital importancia para el hombre. Para seguir gozando de dichos servicios se deberán tomar medidas al respecto, como el implementar períodos de veda y organizar grupos de vigilancia para que puedan estar pendientes de las especies y se puedan proteger, además de regular su captura. Dichas acciones deberán ser realizadas por una participación conjunta de propietarios privados, ejidatarios, comuneros y el gobierno municipal.

El agua es el servicio ambiental más importante que tenemos y el que menos protegemos. Se deberá crear consciencia de su importancia, al igual que mejorar la gestión de dicho servicio ambiental, regulando así su uso y evitando desechar basura a la laguna o contaminádola con otras sustancias. Los propietarios privados, los ejidatarios y comuneros, así como el gobierno municipal deberán realizar dichas acciones.

Para que sigamos contando con un filtro biológico que retenga y procese los contaminantes contenidos y se siga teniendo una producción de oxígeno y captura de dióxido de carbono, se deberá reforestar la zona más necesitada del manglar y analizar periódicamente la calidad del agua, así como la preservación y protección de la misma. Dichas acciones se deberán realizar por propietarios privados, ejidatarios y comuneros, instituciones de investigación, universidades y concesionarios. 
Por último, existen acciones complementarias que serán realizadas por concesionarios, gobierno municipal, habitantes de los municipios, etc. y que son necesarias para el programa de pago por servicios ambientales, aunque no obtengan ningún beneficio monetario por hacerlo.

Los concesionarios podrían aportar acciones básicas como la vigilancia del manglar y realizar programas para que la población conozca la importancia de preservarlo, para los servicios ambientales de protección contra inundaciones, control de la erosión, protección de la diversidad, protección del suelo y en la producción de oxígeno y captura de $\mathrm{CO}_{2} \mathrm{CO}_{2}$.

El gobierno municipal y los habitantes de los municipios realizarán acciones complementarias en servicios ambientales como belleza escénica con la colocación de botes en la zona de manglar y recolección periódica de basura.

Otros servicios ambientales en los que deberá realizar acciones complementarias el gobierno municipal son: la crianza de especies, protección a la diversidad, producción de alimentos y provisión de agua.

\subsection{Identificación de los beneficiados por el pago}

Es necesario "identificar claramente quienes deberían beneficiarse por el pago, es decir, a quien debería pagarse y porqué. Estos beneficiados por el pago son quienes deberían llevar a cabo las acciones de prevención, conservación y/o restauración necesaria para lograr los objetivos del programa" (GARCÍA, 2018b, p. 41-62).

Para poder llevar a cabo lo anterior y poder identificar quienes deberían pagar y porqué, al igual que llevar a cabo las acciones de preservación, conservación y/o restauración, "es conveniente agrupar a los beneficiarios por sectores, ordenándolos por importancia, es decir, priorizando a aquéllos en una situación más vulnerable y, por lo tanto, a los que se considera necesario dar prioridad en el pago. Éste es uno de los aspectos centrales y más complicados en un programa de este tipo" (GARCÍA, 2018b, p. 41- 62).

Los beneficiados por el pago de los servicios ambientales en la laguna de Sontecomapan se dividen en tres grupos:

- el primer grupo engloba a personas con terrenos con manglar de los municipios cercanos a la laguna y se dividen en ejidos y comunidades y en posesionarios;

- el segundo grupo un equipo conformado por los propietarios, ejidos, 
comunidades y posesionarios para la protección de la zona de crianza, refugio, protección de la biodiversidad y alimentación de especies, que aseguran la producción de alimentos y

- el tercer grupo está conformado por los pescadores de la zona.

\subsection{Definición de acciones complementarias}

Los concesionarios podrían aportar acciones básicas como vigilancia del manglar y realizar programas para que la población conozca la importancia de preservarlo, para los servicios ambientales de protección contra inundaciones, control de la erosión, protección de la diversidad, protección del suelo y en la producción de oxígeno y captura de $\mathrm{CO}_{2} \mathrm{CO}_{2}$.

El gobierno municipal y habitantes de los municipios realizarían acciones complementarias en servicios ambientales como "belleza escénica" en colocación de botes en la zona de manglar y recolección periódica de basura.

Otros servicios ambientales en los que deberá realizar acciones complementarias el gobierno municipal son: crianza de especies, protección a la diversidad, producción de alimentos y provisión de agua.

\subsection{Identificación de los "obligados" a pagar y del monto del pago}

Este paso consiste en determinar quienes deberían pagar los servicios ambientales de acuerdo a la información relativa a los beneficiados expuesta previamente y cuánto deberían pagar. Asignar valores monetarios a recursos naturales, en particular para obtener valores de no uso, es complicado y además riesgoso ya que por lo común no se cuenta con todos los elementos para la valoración y se tiende a subvaluar todo el ecosistema. Asignar valores para usos directos e indirectos de los recursos y servicios del ecosistema es de suma importancia para medir parcialmente los beneficios económicos derivados de estos (CALDERÓN Y ABURTO, 2009, p. 1-6). Los valores de uso indirecto se refieren a los beneficios que recibe la sociedad a través de los servicios de los ecosistemas y de las funciones del hábitat. A diferencia del valor de uso directo, el indirecto generalmente no requiere del acceso físico del usuario al recurso natural, pero sí de la presencia del recurso en buenas condiciones. Los valores de uso activo indirecto se pueden clasificar en: valores ambientales y valores ecosistémicos. Entre los servicios ambientales destacan: el filtrado de 
aguas residuales, la protección contra tormentas y el funcionamiento del ecosistema como criadero de especies pesqueras. Por otra parte, entre los valores ecosistémicos se encuentran la captura de carbono, la fijación de nitrógeno en el suelo y la auto preservación del ecosistema (SANSUJO RIVERA Y WELSH CASAS, 2005, p. 55-68). El valor económico de los manglares con los servicios y productos ambientales incluídos que provee va de 200,000 a 900,000 dólares por hectárea al año (WELLS, 2006, p. 33).

Considerando que la laguna de Sontecomapan cuenta con una extensión de 760 hectáreas de manglar y tomando el valor más bajo de Wells (2006, p. 33), se tendría un valor económico aproximado de 152 , 000,000 dólares por hectárea.

Para poder determinar una cuota que los habitantes de los municipios pagarán, se deberá tomar en cuenta el salario mínimo en pesos mexicanos que es de $\$ 102.68$ y mensual de $\$ 3,121.47$. Si la cuota propuesta fuera de $\$ 40$ mensuales para el pago de servicios ambientales, sólo representaría el $1.28 \%$ del salario de los habitantes.

Para que sea más clara la información se tiene que conocer la cantidad de viviendas particulares habitadas que disponen de agua de la red pública:

- Catemaco: 10,786 viviendas;

- San Andrés Tuxtla: 34,850 viviendas;

- Mecayapan: 3,011 viviendas;

- Soteapan: 7,122 viviendas;

- Hueyapan de Ocampo: 7,550 viviendas (INEGI, 2010).

Respecto a lo anterior tenemos un total de 63,319 viviendas que, al ser multiplicadas por los $\$ 40$, que se les cobraría mensualmente en el recibo de agua, nos da un total de $\$ 2,532,760$, y así, multiplicando la cantidad anterior por los doce meses del año tendremos la siguiente cantidad de dinero:

63,319 viviendas $\times \$ 40$ cobro $=\$ 2,532,760$ mensuales

$\$ 2,532,760$ mensual $\times 12$ meses $=\$ 30,393,120$ anuales

Por otro lado, el servicio ambiental de belleza escénica será pagado, parcialmente, por los habitantes $\mathrm{y}$, por otra parte, por los turistas, a través de una cooperación voluntaria en el momento de la visita.

Debido a que no se tiene el número específico o aproximado de las personas que visitan la laguna de Sontecomapan, se tomarán datos abiertos 
del INAH (2018) donde se tienen registros anuales de las visitas de turistas nacionales e internacionales de todos los museos y zonas arqueológicas del estado de Veracruz. El año pasado en el museo Tuxteco el número total de visitantes en el año fue de 4,158 turistas. En el museo de Tres Zapotes el número total de visitantes en el año fue de 4,244 turistas (INAH, 2018). Se calculará la cantidad de turistas que visitarán la zona al mes y la cantidad que sera recaudada por este grupo.

\section{4,158 turistas $+4,244$ turistas $=8,402$ turistas al año}

Haciendo la suposición de que cada turista hará la cooperación propuesta de $\$ 50$, es posible recaudar la siguiente cantidad de dinero a 1 año:

\section{8,402 turistas al año $\times \$ 50$ cooperación $=\$ 420,100$ al año}

Por último, en el caso de que la captura de carbono entrara en los mercados de carbono, para la laguna de Sontecomapan que cuenta con una extensión de 760 hectáreas, se tiene un total de captura de 172,520 toneladas de carbono.

Si dicha tonelada se paga entre 5 y 10 dólares, tomando el valor más bajo se tendría la siguiente cantidad de dinero al año:

$$
\begin{gathered}
172,520 \mathrm{tC} \times 5 \text { dólares }=862,600 \text { dólares } \\
862,600 \text { dólares } \times \$ 19.03=\$ 16,415,278.00
\end{gathered}
$$

Tomando en cuenta la cantidad de dinero que se recaudará, sólo se podrá pagar totalmente el servicio de protección contra inundaciones. El de belleza escénica será pagado parcialmente, con lo restante del servicio anterior y, otra parte, con la cooperación de los turistas; además, se tendrán los bonos de carbono con el servicio ambiental de producción de oxígeno y captura de $\mathrm{CO} 2$.

La cantidad de dinero que se dispone para el pago de lo mencionado anteriormente es de $\$ 47,228,498$ por año. 


\subsection{1 ¿Sobre quiénes recaerá el pago?}

Los sectores propuestos para que recaiga el cobro de los servicios ambientales son los siguientes:

Sector 1: el primer sector en el que recaerá el pago de los servicios ambietales está conformado por los 297,535 habitantes de los municipios de: Catemaco, San Andrés Tuxtla, Mecayapan, Soteapan y Hueyapan de Ocampo.

Estos habitantes de los cinco municipios de la laguna de Sontecomapan se benefician directamente de la mayoría de los servicios ambientales que este sitio Ramsar ofrece. La propuesta consiste en hacer que los habitantes anteriores realicen el pago a través de una couta mensual que será agregada en sus recibos de agua.

Sector 2: el segundo sector se encuentra conformado por turistas estatales y nacionales e internacionales que visitan la zona de manglar de la laguna de Sontecomapan.

Los habitantes de los municipios y los turistas son los que se benefician directamente de este servicio ambiental. Ellos, al visitar la zona, disfrutan del ecosistema de manglar que tiene gran demanda turística, así mismo del paisaje, la vegetación, la fauna y las actividades que se realizan en el lugar. Los turistas son el grupo que pagará gran parte de este servicio al visitar el lugar y dar una cooperación voluntaria de \$50. Dicha cooperación podrá ser efectuada o depositada en botes que estarán colocados en la zona del embarque, en la playa, en restaurantes y puntos de encuentro para visitas guiadas a los manglares y lagunas.

Se implementarán campañas de comunicación, para que las personas que visiten la zona puedan informarse sobre la importancia de preservar y mantener en buenas condiciones el ecosistema que están visitando; así mismo es necesario hacerles saber, a través de una campaña de comunicación adecuada, lo importante que es su cooperación. En la campaña se les propondría una cantidad a cooperar, aunque la decisión de cuanto pagar por visita es completamente personal.

Sector 3: el tercer sector se encuentra conformado por aquellos involucrados en el mercado de carbono. Existen dos tipos de mercados de carbono: los de cumplimiento regulado y los voluntarios. El mercado regulado es utilizado por empresas y gobiernos que, por ley, tienen que rendir cuentas de sus emisiones de gases de efecto invernadero (GEI). Está regulado por regímenes obligatorios de reducción de carbono, ya sean nacionales, 
regionales o internacionales. En el mercado voluntario, en cambio, el comercio de créditos se produce sobre una base facultativa. Las dimensiones de los dos mercados difieren notablemente (SEEBERG-ELVERFELDT, 2010).

Este sistema o mercado voluntario funciona bajo estándares específicos para el desarrollo de los bonos de reducciones voluntarias. La credibilidad es un aspecto clave en el mercado voluntario de carbono, de modo que el uso de estándares conocidos y aceptados mundialmente es indispensable. En el mercado voluntario las empresas, las organizaciones no gubernamentales, los gobiernos y los particulares pueden adquirir créditos de carbono. Quienes desarrollan proyectos verifican compensaciones por medio de agentes independientes, éstas se denominan "Reducciones de Emisiones Verificadas" (VER por su sigla en inglés) y pueden ser vendidas a personas $\mathrm{u}$ organizaciones que deseen compensar sus emisiones de carbono y a empresas o personas que voluntariamente deseen reducir la llamada huella de carbono que dejan sus emisiones (BARROS ASENJO Y IPINZA CARMONA, 2014, p. 39-60).

Es importante destacar que el objetivo inicial de este mercado no era satisfacer regulaciones, sino tan solo la construcción de una "imagen verde" o de Responsabilidad Social Corporativa (RSC), por lo tanto, las normas son más sencillas y por eso reciben un menor precio. El objetivo de este mercado es la reducción de emisiones, y la calidad es asegurada por rigurosos procedimientos técnicos que, normalmente, son desarrollados por estándares específicos y metodologías procedentes del MDL, y además se aplican normas de verificación aceptadas internacionalmente y garantías de desarrollo sostenible. En este sentido, los sellos de Manejo Forestal Sustentable (MFS), herramientas también voluntarias, tales como FSC21 y CERTFOR22/PECF23, cobran una oportunidad inusitada ya que son aceptados por estos mercados. Los mercados voluntarios se acomodan a transacciones de pequeña escala, cuando empresas desean compensar parte de sus emisiones institucionales o construir una "imagen verde", y también personas informadas que desean compensar sus emisiones por viajes aéreos y desplazamientos en general. En el mercado de carbono los proyectos forestales son más rentables al trabajar con especies nativas que con las no nativas, dado que el valor de los bonos puede ser de US 8 a US 10 la tonelada de $\mathrm{CO} 2$, en tanto que con las no nativas los bonos son pagados en el mercado a la mitad de este valor (BARROS ASENJO Y IPINZA CARMONA, 2014, p. 39-60). 


\section{BASES JURÍDICAS PARA LA ELABORACIÓN DE UN PROGRAMA DE PAGO POR SERVICIOS AMBIENTALES}

"Las bases legales sobre las cuales descansará el programa para su puesta en marcha tendrán relación, necesariamente, con el nivel en el que se encuentren aquéllos sobre los que recaerá el pago" (GARCÍA, 2018b, p. 41-62).

En este caso los servicios ambientales que serán tomados en cuenta en esta primera parte son protección contra inundaciones y belleza escénica, dichos servicios serán pagados por los habitantes de los municipios, encontrando las bases jurídicas en el derecho municipal.

Los pagos se harán de manera voluntaria a través de aportaciones que ya han sido establecidas previamente y se hará la creación de un fondo ambiental.

Existen tres tipos de fondos ambientales: el público puede hacerse de manera directa transfiriendo a unos u otros dinero del presupuesto nacional, o de manera indirecta, mediante la concesión de ventajas, prerrogativas, aportes en especie o subvenciones o como incentivo para las donaciones privadas. Por otro lado, el financiamiento privado es el que proviene, en dinero o en especie, del patrimonio de los particulares y cuando concurren ambas formas de manera simultánea, se dice que el financiamiento es mixto (DE LA CALLE, 2004, p. 21-45).

En este caso, se hará la constitución de un fondo ambiental mixto para que pueda nutrirse tanto de aportaciones públicas como privadas. Es muy importante mencionar que los fondos ambientales son entidades a través de las cuales se implementan mecanismos innovadores de financiamiento. Estos mecanismos empezaron a generalizarse en la década del 90.

Incluyen fondos fiduciarios establecidos por legislaciones especiales, fundaciones, fideicomisos de derecho consuetudinario y sociedades no lucrativas. La mayoría de los fondos ambientales son administrados por consejos de administración que incluyen a representantes tanto del gobierno anfitrión como de organizaciones no gubernamentales. La modalidad que más nos interesa son los fideicomisos, que son un contrato en virtud del cual una o más personas, llamada fiduciante, transmite bienes, cantidades de dinero o derechos, presentes o futuros, de su propiedad a otra persona (física o jurídica) llamada fiduciario, para que esta administre o invierta los bienes en beneficio propio o en beneficio de un tercero, llamado fideicomisario. Los bienes afectados al fideicomiso no corren el riesgo 
comercial del fiduciante ni del fiduciario, puesto que el patrimonio que es objeto del fideicomiso no puede ser perseguido por los acreedores de ninguno de ellos, ni afectado por la quiebra de ambos o de alguno de ellos (FVSA, 2012). "Tienen como objetivo financiar inversiones en apoyo a la conservación y a la protección del ambiente, con particular énfasis en aquellas actividades programadas o incluidas bajo el proyecto de manejo de recursos naturales" (RODRÍGUEZ BECERRA Y ESPINOZA, 2002).

Los fondos ambientales han desempeñado un papel importante para garantizar la conservación de la biodiversidad a largo plazo en todo el mundo a través de la posibilidad de movilizar recursos financieros significativos. En la actualidad, tienen la posibilidad de propiciar el avance en mercados emergentes y recompensar a las comunidades locales a través de una serie de mecanismos que incluyen la compra de créditos de compensación o a través de fondos de arranque de proyectos promisorios. Históricamente, los fondos ambientales han tenido un rol muy importante al asegurar la conservación de largo plazo de la biodiversidad del planeta gracias a su capacidad de movilizar grandes sumas de recursos financieros. Hoy, tienen el potencial de impulsar iniciativas de pago por servicios ambientales mediante una variedad de mecanismos (HERBERT et al., 2010).

Se buscará enlazar esta propuesta de diseño de pago por servicios ecosistémicos con fondos ambientales y fideicomisos que son instrumentos financieros "que buscan brindar recursos financieros para la protección y/o restauración del medio ambiente" (GARCÍA, 2017, p. 267) y que forman parte de los instrumentos económicos.

\subsection{Contrato de pago por servicios ambientales}

Debido a que los pagos por servicios ambientales, ya sea para la biodiversidad, para el secuestro de carbono o para la purificación de agua, implican la obligación de gestionar la tierra en cierta forma y por cierto tiempo a cambio de una compensación, las partes deben celebrar algún tipo de acuerdo. Un acuerdo eficaz garantizará que las partes comprendan tanto sus propios derechos, obligaciones y asignación de riesgos como los de las otras partes. La claridad puede ayudar a reducir la probabilidad de conflictos y fracasos más adelante. El acuerdo más conveniente para los PSA hídricos dependerá de las circunstancias locales. Con anterioridad a la redacción de acuerdos específicos, será preciso desarrollar una estrategia general. Lo esencial es que cada acuerdo de PSA ha de enmarcarse dentro de un plan 
de proyecto más amplio para garantizar que los acuerdos individuales sean complementarios y apoyen el objetivo general (GREIBER, 2010).

Una vez que la estrategia general de PSA ha sido establecida y se ha desarrollado el plan del proyecto, el primer paso en la redacción de acuerdos individuales consiste en determinar las partes a involucrar en la transacción. Hay una amplia gama de opciones disponibles y los detalles del contrato dependerán en gran medida de la naturaleza de las partes. Las transacciones de PSA a menudo implican un único comprador y múltiples vendedores. Otros tipos de relaciones pueden incluir un solo comprador y vendedor o un solo comprador y varios vendedores, agrupados a través de una cooperativa u otra institución que se ocupa de los problemas colectivos (GREIBER, 2010).

Hay, sin embargo, ventajas importantes con respecto a los contratos escritos y vinculantes que deben ser consideradas desde el principio. Los acuerdos escritos consignan los derechos y obligaciones de las partes, reduciendo la posibilidad de malentendidos con respecto a las condiciones originales del acuerdo. Los acuerdos escritos también proporcionan un registro al que se puede hacer referencia en caso de desacuerdo. El proceso de redacción de un acuerdo propicia una mejor comprensión de la naturaleza de los PSA y de los acuerdos comerciales. Por último, el acto mismo de firmar un documento jurídicamente vinculante puede reforzar la importancia de las obligaciones en el documento, reduciendo quizás el riesgo de incumplimientos posteriores (GREIBER, 2010).

La esencia del contrato está contenida en las condiciones que establecen los derechos y obligaciones del vendedor y el comprador. Ahí se especifican las acciones que cada parte debe llevar a cabo para cumplir con el acuerdo, así como las reclamaciones concretas de una parte en contra de la otra. El acuerdo debe indicar claramente las expectativas del comprador y el vendedor.

Otro punto importante en cualquier contrato de pago es la duración. Si bien los proyectos de conservación subvencionados pueden demostrar éxito temporal, la terminación de las subvenciones puede dar lugar a que los propietarios de tierras regresen a sus anteriores prácticas de gestión, en detrimento del hábitat que habían conservado. Desde la perspectiva del comprador, un contrato más largo podría ser, por lo tanto, preferible. Sin embargo, consideraciones prácticas, tales como la financiación disponible, los cambios políticos en la administración y el riesgo de inflación, pueden 
hacer de la estructura de pagos fijos a largo plazo una inversión arriesgada en vez de segura (GREIBER, 2010).

\section{CONCLUSIONES}

1. Es posible que con la aplicación de un pago por servicios ambientales se conserven las hectáreas de manglar de la laguna de Sontecomapan y se empiece a hacer conciencia en los pobladores y en todos los que se benefician del manglar de su importancia.

2. La laguna de Sontecomapan no goza de ninguna protección específica, más allá de la genérica que establece la legislación aplicable, para evitar la tala de árboles de mangle o el robo de especies de la zona, a pesar de ser un sitio Ramsar.

3. A través de incentivos se le puede pagar a los propietarios de los terrenos con manglar para que éstos realicen prácticas de manejo sostenible en sus tierras y se pueda proteger y conservar de una mejor forma, así mismo preservando los servicios que provee.

4. Con la aplicación de este programa de pago por servicios ambientales se podría hacer realidad el tercer escenario planteado en este trabajo, llamado optimista, en el que se espera la recuperación de hectáreas del manglar de Sontecomapan.

5. Con la aplicación de este programa se podrá pagar en un inicio el servicio de protección contra inundaciones, servicio que es muy importante para los pobladores y del cual generalmente desconocen su valor y las ventajas que les brinda.

6. Otro de los servicios ambientales que se propone pagar es el de belleza escénica, donde los beneficiados directos son los turistas nacionales e internacionales que visitan el manglar de Sontecomapan y son muy importantes para esta propuesta.

7. El servicio de captura de carbono entrará en los mercados de carbono y se podrá generar un cantidad de dinero que se empleará para pagarle a las comunidades, ejidos, propietarios privados, pescadores, etc. por las acciones que realizan para seguir preservando los servicios ambientales que provee la laguna.

8. Esta propuesta de pago por servicios ambientales nos brinda una serie de beneficios a todos al poder seguir manteniendo y protegiendo el manglar, seguir generando servicios ambientales de los que se benefician los habitantes de la cuenca, de los municipios, del estado, del 
país, turistas, empresas, pescadores, etc., posibilitando la recuperación de manglar y atrayendo más turistas que promueven la economía del lugar.

\section{REFERENCIAS}

BARROS ASENJO, S.; IPINZA CARMONA, R. El mercado del carbono. In: BARROS, S.; IPINZA, R. (Eds.). El cambio climatico, los bosques y la silvicultura. Santiago: Infor, 2014. p. 39-60.

CALDERÓN, C.;ABURTO, E. E.: El valor de los manglares. BioDiversitas, v. 82, p. 1-6, 2009.

CONAGUA - COMISIÓN NACIONAL DEL AGUA. Caracterización fluvial e hidráulica de las inundaciones en México: cna-sgt-gasir-09/2014. Organismo de cuenca X, Golfo de México, 2014.

DE LA CALLE, H. Financiamiento político: público, privado, mixto. In: GRINER, S.; ZOVATTO, D. De las normas a las buenas prácticas: el desafío del financiamiento político en América Latina. San José: OEA/ IDEA, 2004. p. 21-45.

ESTEROS, P. Ficha Informativa de los Humedales de Ramsar (FIR). Wetlands, 1-12, 2003. Disponible en: https://doi.org/10.1007/s13398-0140173-7.2. Acceso en: 2 mar. 2019.

FREGOSO, A. La oferta y el pago de los servicios ambientales hidricos: una comparación de diversos estudios. Gaceta Ecológica, n. 78, p. 29-46, 2006.

FVSA - FUNDACIÓN VIDA SILVESTRE ARGENTINA. Taller sobre fondos ambientales: experiencias en América Latina y el Caribe y su posible aplicación para la conservación de la biodiversidad en Argentina. 2012. Disponible en: <https://d2qv5f444n933g.cloudfront.net/downloads/ reportetaller_fondos_ambientales_1.pdf>. Acceso en: 8 jul. 2019.

GARCÍA LÓPEZ, T. Building a methodology for the design of an environmental services payment programme for the Mangroves of Mexico. Open Journal of Ecology, v. 8, n. 3, p. 147-165, 2018 a.

GARCÍA LÓPEZ, T. Hacia una metodología para el diseño de programas de pago por servicios ambientales en manglares en México. Cuadernos de Biodiversidad, n. 54, p. 41-62, 2018 b.

GARCÍA LÓPEZ, T. Instrumentos económicos en materia ambiental: bases jurídicas para el diseño de políticas públicas. México: Fundación Jacobea, 2017. p. 267. 
GREIBER, T. (Ed.). Pagos por servicios ambientales: marcos jurídicos e institucionales. Gland: UICN, 2010.

INAH - INSTITUTO NACIONAL DE ANTROPOLOGÍA E HISTORIA. Red de museos y zonas arqueológicas del INAH. Disponible en: $<$ https:// www.inah.gob.mx/museos>. Acceso en: 11 dic. 2018.

INEGI - INSTITUTO NACIONAL DE ESTADÍSTICA, GEOGRAFÍA E INFORMÁTICA. Censo General de Población y Vivienda: resultados definitivos. Aguascalientes: Inegi, 2010.

LARA-DOMÍNGUEZ, A. L. et al. Caracterización del sitio de manglar Sontecomapan. México, DF: Conabio, 2009.

MARTÍNEZ CRUZ, D. A. et al. Disposición de los productores forestales de la región Izta-Popo a aceptar pagos por mantener los servicios ambientales hidrológicos. Tropical and Subtropical Agroecosystems, v. 12, n. 3, p. 549$556,2010$.

OLGUÍN, E. J., HERNÁNDEZ, M. E.; SÁNCHEZ GALVÁN, G. Contaminación de manglares por hidrocarburos y estrategias de biorremediación, fitorremediación y restauración. Revista Internacional de Contaminación Ambiental, v. 23, n. 3, p. 139-154, 2007.

PEREVOCHTCHIKOVA, M.; OCHOA TAMAYO, A. M. Avances y limitantes del programa de pago de servicios ambientales hidrológicos en México. Rev. Mex. Cien. For., v. 3, n. 10, p. 89-112, 2012. Disponible en: $<$ http://www.scielo.org.mx/pdf/remcf/v3n10/v3n10a8.pdf $>$. Acceso en: 20 mar. 2019.

RAMSAR. Criterios para Sitios Ramsar. Convención sobre humedales, 1971. Disponible en: <http://www.ramsar.org/sites/default/files/documents/library/ramsarsites_criteria_sp.pdf $>$. Acceso en: 3 feb. 2019.

RAMSAR. Convención Relativa de los Humedales de Importancia Internacionel Espciealmente como Hábitat de Aves Acuáticas, 1987. Disponible en: <https:/www.ramsar.org/sites/default/files/documents/library/ current_convention_s.pdf $>$. Acceso en: 3 feb. 2019.

SANSURJO RIVERA, E.; WELSH CASAS, S. Una descripción del valor de los bienes y servicios ambientales prestados por los manglares. Gaceta Ecológica, v. 74, p. 55-68, 2005.

RODRÍGUEZ BECERRA, M., y ESPINOZA, G. Gestión ambiental en América Latina y el Caribe: Evolución, tendencias y principales prácticas. EUA: Banco Interamericano de Desarrollo, 2002. 
SECRETARÍA DE LA CONVENCIÓN DE RAMSAR. Manual de la Convención de Ramsar. 6 ed. Gland: Secretaría de la Convención de Ramsar, 2013. Disponible en: <https://www.ramsar.org/sites/default/files/ documents/library/manual6-2013-sp.pdf>. Acceso en: 19 ene. 2019.

SEEBERG-ELVERFELDT, C. Las posibilidades de financiación del carbono para la agricultura, la actividad forestal y otros proyectos de uso de la tierra en el contexto del pequeño agricultor. Roma: Organización de las Naciones Unidas para la Agricultura y la Alimentación, 2010.

SEFIPLAN. Estudios regionales para la planeación; región Papaloapan. México: Gobierno del Estado, 2011.

TOLEDO, V. M.; GUEVARA, S.; HERNÁNDEZ, J. Un posible método para evaluar el conocimiento ecológico de los hombres de campo. In: LOT HELGUERAS, C. A. et al. Problemas biológicos de la región de Los Tuxtlas, Veracruz. México, DF: Universidad Nacional Autónoma de México, 1972. p. 199-237.

HERBERT, T. et al. Fondos ambientales y pagos por servicios ambientales: proyecto de capacitación de RedLAC para fondos ambientales. Rio de Janeiro: RedLAC, 2010.

WELLS, S. In the front line: shoreline protection and other ecosystem services from mangroves and coral reefs. Cambridge, UK: UNEPA World Conservation Monitoring Centre, 2006. p. 33.

Artículo recibido en: 10/06/2019. Artículo aceptado en: 08/07/2019.

\section{Cómo citar este artículo (ABNT):}

GARCÍA LÓPEZ, T.; GUDIÑO ANAYA, M. L. Propuesta y bases jurídicas para la elaboración de un programa de pago por servicios ambientales para la Laguna de Sontecomapan, México. Veredas do Direito, Belo Horizonte, v. 16, n. 35, p. 37-61, maio/ago. 2019. Disponível em: <http://www. domhelder.edu.br/revista/index.php/veredas/article/view/1558>. Acesso em: dia mês. ano. 